\title{
Polyserase-1/TMPRSS9 induces pro-tumor effects in pancreatic cancer cells by activation of pro-uPA
}

\author{
TANIA FONTANIL ${ }^{1,2}$, YAMINA MOHAMEDI ${ }^{1,2}$, MANUEL M. ESTEBAN $^{2,3}$, \\ ALVARO J. OBAYA ${ }^{1,3}$ and SANTIAGO CAL ${ }^{1,2}$ \\ ${ }^{1}$ Department of Biochemistry and Molecular Biology, ${ }^{2}$ University Institute of Oncology of Principado de Asturias (UOPA), \\ and ${ }^{3}$ Department of Functional Biology-Physiology, University of Oviedo, 33006 Oviedo, Asturias, Spain
}

Received January 9, 2014; Accepted March 13, 2014

DOI: $10.3892 /$ or.2014.3146

\begin{abstract}
Polyserase-1/TMPRSS9 is a type II transmembrane serine protease showing a complex molecular architecture characterized by the presence of three tandem serine protease domains in its amino acid sequence. This protease is widely expressed in mouse and human tissues, however, its functional significance is unknown in both normal and pathological conditions. In the present study, we evaluated the possible role of polyserase-1 in cancer progression. First, we showed that polyserase-1 increased the invasive capacities of PANC-1 and SK-PC-3 pancreatic cancer cells. Moreover, the presence of polyserase-1 enhanced anchorage-independent growth and diminished the adhesion capability of PANC-1 cells to different extracellular matrix components. These effects were mediated by the efficient conversion of pro-uPA to active uPA and high phosphorylation levels of ERK detected in the PANC-1 cells expressing exogenous polyserase-1. Collectively, our data suggest that polyserase-1 may be involved in cancer progression and, similarly to what has been proposed for the closely related serine proteases matriptase and TMPRSS4, inhibition of TMPRSS9 activity may contribute to the inhibition of tumor growth.
\end{abstract}

\section{Introduction}

The tightly controlled proteolytic turnover of extracellular matrix (ECM) components in the immediate vicinity of the cell has a significant influence in the development and maintenance of tissue homeostasis (1). Proteolytic events within this pericellular microenvironment are mainly executed by extracellular or cell-surface metalloproteases and serine proteases (2). Among them, the type II transmembrane serine proteases (TTSPs), are membrane-anchored serine proteases

Correspondence to: Dr Santiago Cal, Department of Biochemistry and Molecular Biology, University of Oviedo, 33006 Oviedo, Asturias, Spain

E-mail: santical@uniovi.es

Key words: serine protease, polyserase, TMPRSS9, extracellular matrix, PANC-1, anchorage-independent growth involved in relevant biological functions (3). Thus, enteropeptidase is produced by the duodenum and its function is essential to digest food efficiently (4). Corin contributes to blood pressure control through activation of atrial natriuretic peptide (5). Matriptase-2 participates in iron homeostasis (6), and hepsin is implicated in the process of hearing (7). These and other examples (8) illustrate the relevance of TTSPs in normal physiological processes.

The activities of the TTSPs also underlie different human disorders, including cancer. In fact, these proteases can cause an active pericellular degradation to breach tissue barriers thereby facilitating invasion of surrounding tissues (9). Moreover, this pericellular proteolysis may also trigger the activation of growth factors that promote tumor cell proliferation and migration (10). However, TTSPs can also display antitumor effects. For instance, $H P N$, the gene encoding for human hepsin, is one of the most highly upregulated in prostate cancer, and this enzyme is exclusively detected in the surface of prostate carcinoma cells (11). In contrast, low expression levels of hepsin correlate with shorter patient survival in hepatocellular carcinoma (12). Similarly, expression of matriptase was found to be markedly upregulated in different tumors of epithelial origin, including breast, lung or pancreatic carcinomas (13). However, a significant decrease in its expression was found in gastrointestinal tumors (14). DESC1 serine protease exhibits reduced expression levels in head and neck carcinomas in comparison with matched normal tissues (15), while its exogenous expression confers pro-tumor effects to MDCK cells and it was found to be upregulated in kidney, liver, breast and brain tumors (16). Additionally, different studies have highlighted the functions of different TTSPs as regulators of cellular signaling pathways and revealed the biochemical mechanisms underlying their pro-tumor or antitumor effects (9).

Despite the increased understanding of the functional relevance of TTSPs, some members of this family have been characterized at the structural level while their roles in normal or pathological processes remain unknown. This is the case of polyserase-1 or transmembrane protease, serine 9 (TMPRSS9), a TTPS widely distributed in human and mouse tissues $(17,18)$. Structurally, this enzyme shows a complex molecular architecture containing three tandem serine-protease domains called serase-1, serase-2 and serase-3 (17). The third domain 
is predicted as catalytically inactive due to the presence of an alanine residue instead of the serine residue present in the active site of this type of enzyme. Serase-1B is a splice variant of TMPRSS9 containing only one serase domain which efficiently activates pro-uPA (18). Active uPA converts plasminogen to plasmin, an enzyme that cleaves different ECM components and activates latent collagenases. These effects contribute to the invasiveness of tumor cells of different origin, including pancreatic carcinomas. In fact, uPA exhibits marked overexpression in this type of tumor, and its detection provides prognostic information (19). Different reports have emphasized the pro-tumor activities of different TTSPs in pancreatic cancer $(9,20,21)$. Activation (22) or upregulation (23) of pro-uPA may underlie the pro-tumor effects caused by these serine proteases. In the present study, we investigated the effects of the exogenous expression of polyserase- 1 in pancreas-derived tumor cells $(24,25)$. Collectively, our data suggest that polyserase-1 exerts pro-tumor effects through a mechanism involving activation of pro-uPA and phosphorylation of ERK.

\section{Materials and methods}

Cell lines and culture conditions. The cell line PANC-1 was purchased from Cell Lines Service and the SK-PC-3 cell line was kindly provided by Dr Francisco Real (National Cancer Research Centre, Madrid, Spain). Cells were routinely maintained at $37^{\circ} \mathrm{C}$ in $5 \% \mathrm{CO}_{2}$ in Dulbecco's modified Eagle's medium (DMEM) supplemented with $10 \%$ fetal bovine serum (FBS).

Transfection and western blot analysis. The cell lines were transfected with the plasmid pcDNA3-FLAG-poly1, which contains the complete sequence of polyserase-1 and a FLAG epitope at the amino terminal region (17). Cells transfected with an empty pcDNA3 vector were employed as a control. Transfections were performed using the Lipofectamine system (Invitrogen) following the manufacturer's recommendations. Cells were selected in the presence of G-418 (Sigma-Aldrich) at $400 \mu \mathrm{g} / \mathrm{ml}$. For western blot analysis, the proteins were separated on SDS/PAGE, and blotted onto PVDF membranes. Detection of exogenous polyserase-1 was performed using and an anti-FLAG antibody (Sigma Aldrich), and the H-140 antibody from Santa Cruz Biotechnology was used to detect uPA. Antibodies to detect p-ERK and ERK were from Cell Signalling Technology.

$q R T-P C R$. Total RNA from cell cultures was obtained using the RNeasy Mini kit (Qiagen) following the manufacturer's recommendations. For cDNA synthesis, $2 \mu \mathrm{g}$ of RNA was used as a template and the system ThermoScript RT-PCR (Invitrogen) was employed following the protocol recommended by the manufacturer. cDNA was then used as a template for quantitative PCR using TaqMan probe HS00917112_m1, TaqMan Master Mix reagent and the detection system 7300 Real-Time PCR system (Applied Biosystems). Expression of human $\beta$-actin was employed as endogenous control. Data analysis was performed using the computer program ABI PRISM 7900HT, and RQ Manager 1.2 (Applied Biosystems).
Cell invasion assay. In vitro invasion potential was evaluated using 24-well Matrigel-coated invasion chambers with a $8-\mu \mathrm{m}$ pore size (BD Biosciences). Cells $\left(6 \times 10^{4}\right)$ were allowed to migrate for $24 \mathrm{~h}$ using $10 \%$ FBS as a chemoattractant. Cells that reached the lower surface were fixed and stained with crystal violet. Three independent experiments were performed with triplicates for each condition. Cells were counted in eight randomly selected microscopic fields.

Soft agar colony formation. Anchorage-independent growth was examined using CytoSelect ${ }^{\mathrm{TM}}$ 96-Well In Vitro Tumor Sensitivity Assay kit (Cell Biolabs) following the manufacturer's instructions. Cell density was $6 \times 10^{3}$ cells $/ \mathrm{ml}$, and assays were performed for 8 days at $37^{\circ} \mathrm{C}$ and $5 \% \mathrm{CO}_{2}$ using 24-well Ultra-low attachment plates (Costar). Colonies were examined and photographed with a Leica microscope EZ 2.0 (Leica Microsystems).

Cell adhesion assay. The adhesion capacity of the different cell lines was analyzed using a colorimetric ECM Cell Adhesion Array kit (EMD Biosciences) following the manufacturer's instructions. Briefly, $2 \times 10^{5}$ cells were incubated for $2 \mathrm{~h}$. The cells were then lysed, and the absorbance was measured at $485 \mathrm{~nm}$ using a Synergy H4 Hybrid reader. All data are the mean of three independent experiments.

Statistical analysis. Statistical analysis was performed using the GraphPad Prism 5.0 software. Data are represented as means \pm SE. The occurrence of significant differences was determined with the Student's-Welch t-test. P-values $<0.05$ were considered to indicate statistically significant differences.

\section{Results}

Exogenous expression of TMPRSS9/polyserase-1 in pancreatic cancer cells. To examine whether polyserase-1 affects the tumorigenic phenotype of pancreatic cancer cells, we employed two cell lines, PANC-1 and SK-PC-3, to perform further functional analysis. These cell lines do not endogenously express TMPRSS9 as assessed by qRT-PCR; therefore, cells were transfected using the full-length cDNA for polyserase-1 (17) (Fig. 1A). Expression of exogenous polyserase-1 was assessed using qRT-PCR and western blot analysis by detection of the FLAG epitope at the amino-terminal region of the recombinant protein (Fig. 1A). Cells that stably expressed TMPRSS9, referred to as PANC-1/TMPRSS9 and SK-PC-3/TMPRSS9, respectively, were chosen for further evaluation of potential phenotypic changes. PANC-1 and SK-PC-3 cells transfected with an empty vector, PANC-1c and SK-PC-3c, respectively, did not show immunoreactivity for the antibodies against FLAG epitope and were used as negative controls.

Polyserase-1 increases invasive properties of pancreatic cancer cells. Invasive capacity of the PANC-1/TMPRSS9 and SK-PC-3/TMPRSS9 cells was examined using Matrigel-coated invasion chambers (Fig. 1B). Average cell numbers penetrating the Matrigel per field were 312 in the case of PANC-1/TMPRSS9 cells and 62 in the case of SK-PC-3/TMPRSS9 cells. However, only 102 PANC-1c and 10 SK-PC-3c invasive cells per field were observed in this assay (Fig. 1B). These data indicated that 

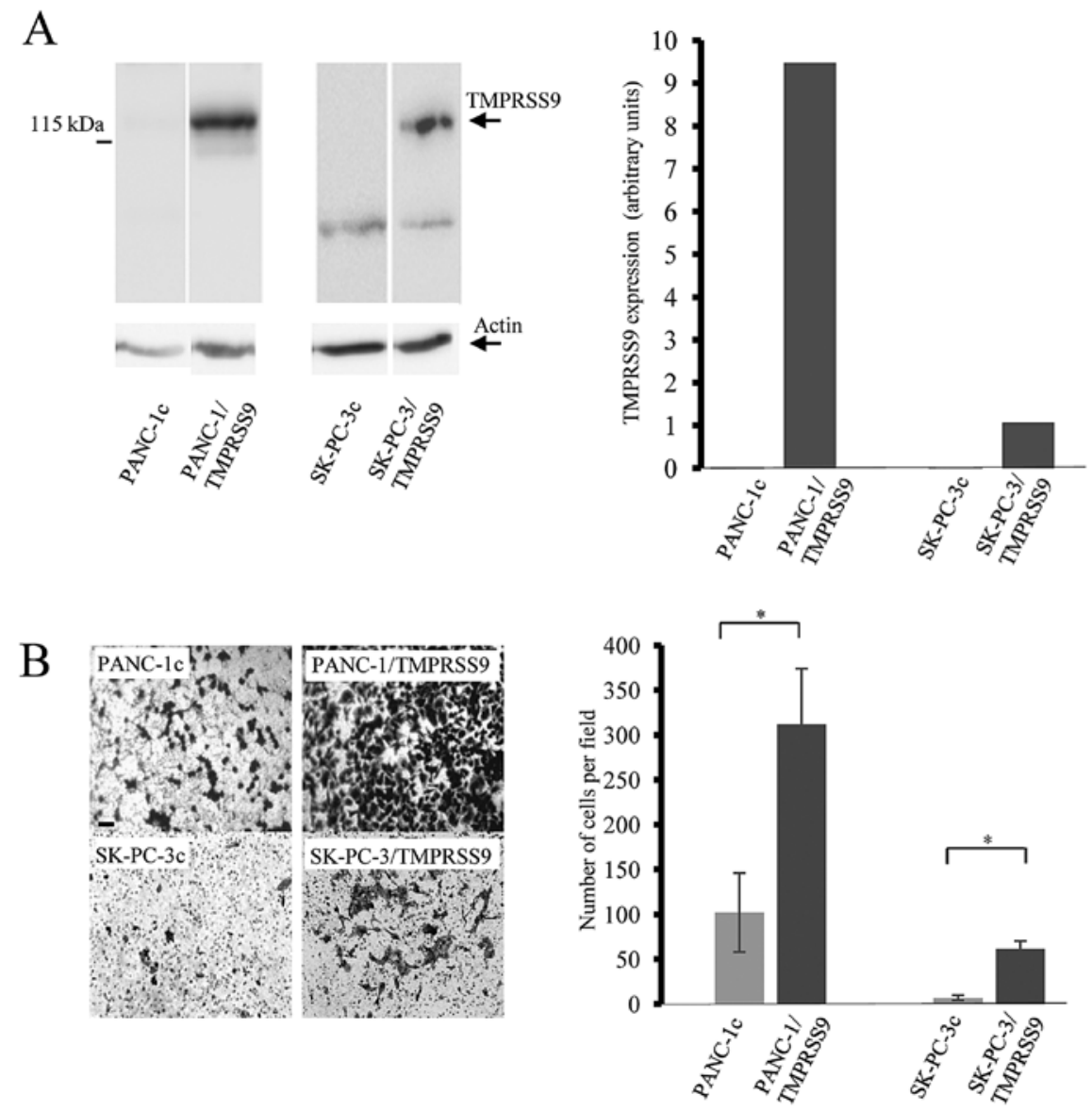

Figure 1. Selection of PANC-1 and SK-PC-3 stable transfectants and invasion assay. (A) Left: western blot analysis of PANC-1 and SK-PC-3 cells expressing exogenous polyserase-1/TMPRSS9, indicated as PANC-1/TMPRSS9 and SK-PC-3/TMPRSS9, respectively. PANC-1c and SK-PC-3c indicate the control cells, transfected with an empty vector. The molecular weight marker is indicated on the left. Actin was used as a loading control. Right: qRT-PCR for mRNA expression levels of TMPRSS9 in the indicated cells. (B) Cell invasion assay using Matrigel-coated invasion chambers. Left: representative microscopic images of the indicated cells. Scale bar, $50 \mu \mathrm{m}$. Right: cells that reached the lower surface were counted, and the numbers are graphically represented. ${ }^{*} \mathrm{p}<0.05$.
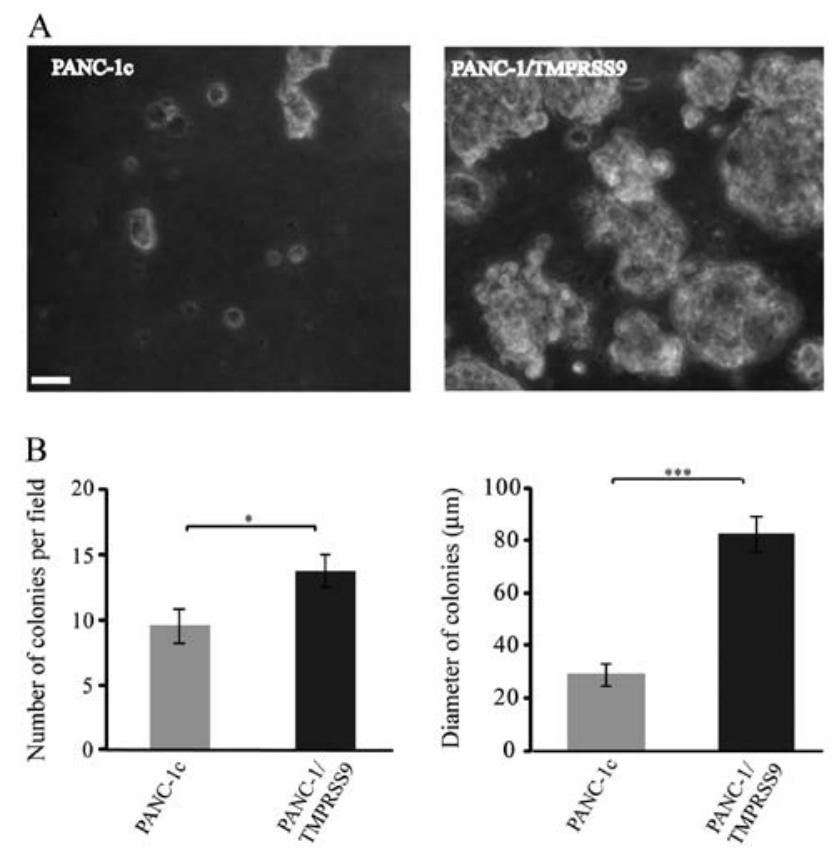

Figure 2. Polyserase-1/TMPRSS9 increases anchorage-independent growth of PANC-1 cells. (A) Representative microscopic images of colonies formed by PANC-1c and PANC-1/TMPRSS9 cells. Scale bar, $50 \mu \mathrm{m}$. (B) Graphical representation of the number and diameter of the colonies. ${ }^{*} \mathrm{p}<0.05,{ }^{* * *} \mathrm{p}<0.005$. the presence of polyserase-1 markedly increases the invasive capacity of both PANC-1 and SK-PC-3 cells.

Polyserase-1 enhances anchorage-independent growth of PANC-1 cells. Results obtained from invasive assays prompted us to perform new cell-based assays using PANC-1, a cell line commonly employed as an in vitro model of pancreatic cancer for tumorigenicity studies since its invasive and proliferative phenotypes have been widely characterized (24). Thus, we carried out a soft-agar colony formation assay to compare the PANC-1/TMPRSS9 and PANC-1c cells and, as shown in Fig. 2A, the colonies formed by cells expressing polyserase-1 were more numerous (14 vs. 9) and much larger (82 $\mu \mathrm{m}$ vs. $30 \mu \mathrm{m}$ in diameter) than those formed by the PANC-1c cells after 8 days of growth (Fig. 2B). These data are also consistent with the hypothesis that polyserase- 1 acts a pro-tumor protease.

Presence of polyserase-1 activates pro-uPA and increases phosphorylation levels of ERK. Different mechanisms have been proposed to underlie the pro-tumor properties associated with the activities of TTSPs, including activation of pro-uPA or modulation of the ERK signaling pathway (23). We examined whether exogenous polyserase- 1 also affects these mechanisms in PANC-1 cells. Toward this end, we performed 


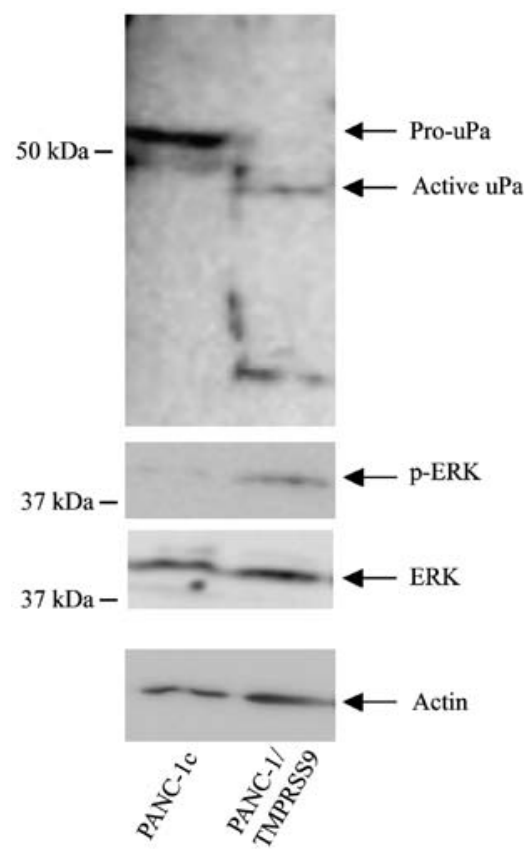

Figure 3. Western blot analysis showing pro-uPA activation and levels of phosphorylated ERK (p-ERK) and total ERK (ERK) in PANC-1c and PANC-1/TMPRSS9 cells. Molecular weight markers are indicated on the left. Actin was used as a loading control.

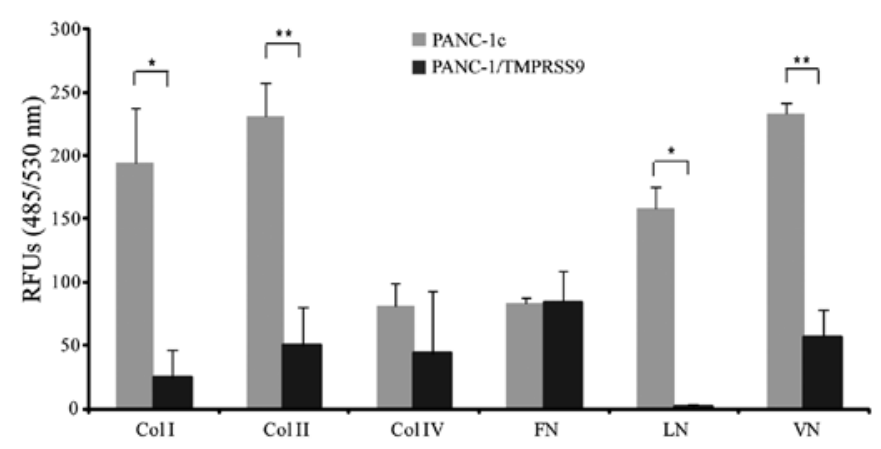

Figure 4. Presence of polyserase-1/TMPRSS9 modifies the adhesion profile of PANC-1 cells to different ECM components. Col I, type I collagen; Col II, type II collagen; Col IV, type IV collagen; FN, fibronectin; LN, laminin; VN, vitronectin. ${ }^{*} \mathrm{p}<0.05,{ }^{* *} \mathrm{p}<0.01$.

western blot analysis that revealed a significant activation of pro-uPA and high levels of phosphorylated ERK (p-ERK) in the PANC-1/TMPRSS9 but not in the PANC-1c cells (Fig. 3). These results suggest that TMPRSS9 may contribute to induce cancer cell invasion by enhancing the activation of survival pathways in tumor cells.

Polyserase-1 alters the adhesive properties of PANC-1 cells. To evaluate whether polyserase-1 alters adhesion of PANC-1 cells to different ECM substrates, we performed a fluorometric assay using PANC-1/TMPRSS9 and PANC-1c cells. The results clearly indicated that the presence of polyserase-1 reduced the capacity of PANC-1 cells to bind to collagens as well as to vitronectin, and completely abolished adherence to laminin (Fig. 4). However, polyserase-1 did not alter the binding capacity of PANC-1 cells to fibronectin. Overall, these results indicated that polyserase-1 induced changes in cell adhesion which eventually leads to increased tumor progression.

\section{Discussion}

Proteolytic enzymes have been traditionally associated with tumor progression. In fact, proteases produced by cancer cells contribute not only to destroy the ECM barrier but also to actively release growth factors to control cell survival. However, recently, a growing number of proteases have also been associated with tumor-protective functions (26). One future challenge includes the identification of the precise functions of the proteolytic enzymes related to tumorigenesis. In this regard, we evaluated the potential contribution of polyserase-1/TMPRSS9 to tumorigenesis employing cellbased assays. This unique enzyme belongs to the TTSP family of membrane-anchored serine proteases and is widely distributed in human and mouse tissues, yet its functional relevance is largely unknown (18). Polyserase- 1 possesses three serine-protease domains in tandem each of which shows a high degree of identity with other members of the TTSP family, particularly with those belonging to the matriptase subfamily (17). Different TTSPs such as TMPRSS4, initially referred to as TMPRSS3 (20) and matriptase (21) have been shown to display pro-tumor activity in various types of tumors, including pancreatic carcinoma, one of the most aggressive cancers. In the present study, we demonstrated that polyserase-1 also acts as a pro-tumor protease. Thus, the presence of this enzyme increased the invasive properties of both SK-PC-3 and PANC-1 pancreatic cancer cells using Matrigelcoated invasion chambers. Moreover, different reports have illustrated the usefulness of PANC-1 to examine the effect of the overexpression (27) or downregulation (28) of particular genes on anchorage-independent growth, a hallmark of malignant transformation. Using similar approaches, we also found that the presence of polyserase- 1 considerably enhanced this effect in PANC-1 cells.

Serase-1B, a spliced variant of polyserase-1 containing one serine protease domain, is able to activate pro-uPA (18), a serine protease largely involved in tumor progression. PANC-1 cells expressing polyserase-1 were also found to exhibit significant pro-uPA activation when compared to PANC-1 control cells. Furthermore, the presence of polyserase- 1 also increased levels of ERK phosphorylation in this cell line. Similar effects have been previously associated to pro-tumor TTPSs. For example, TMPRSS4 upregulates uPA expression and activation in the DU145 prostate cancer and the NCI-H322 lung cancer cell lines by a mechanism involving modulation of the ERK signaling pathway (23). Matriptase is also able to activate pro-UPA in the ovarian cancer cell line HRA (29) and the monocytic leukemia cell line THP-1 (22). On the other hand, induction of invasive and migratory capacities of PANC-1 cells has been linked to activation of ERK by phosphorylation (30). Collectively, our data suggest that TMPRSS9 exerts pro-tumor effects on PANC-1 cancer cells overcoming apoptotic signals induced in anchorage-dependent cells when detaching from the surrounding ECM.

ECM components also affect the adhesive properties of tumor cells. In the case of PANC-1 cells, adhesion capacity to type I, II and IV collagens, laminin, fibronectin and vitronectin 
has been previously assessed (24). Our data indicated that polyserase-1 reduces the adhesion capacity of PANC-1 cells to collagens, laminin and vitronectin which may contribute to the promotion of cell migration and invasion (31). In reference to other TTSPs, overexpression of matriptase and TMPRSS4 was also found to alter the adhesion profile of tumor cells to ECM components thereby contributing to facilitate their invasive and metastatic properties $(32,33)$.

In summary, in the present study, we provide initial evidence suggesting that polyserase-1 induces pro-tumor activities in pancreatic cancer cells and that UPA and ERK may participate in these processes. Consequently, polyserase-1 may be involved in tumor progression, and inhibition of its activity could contribute to prevent progression of this malignant tumor. Further exhaustive in vivo functional studies will help to shed light on the participation of polyserase-1 in tumorigenesis. In this regard, generation of mice lacking TMPRSS9 may be a useful tool to understand the precise role of this polyserase-1 not only in pancreatic carcinoma but also in tumors from different origins.

\section{Acknowledgements}

We thank Dr Carlos Lopez-Otin for his comments and suggestions. The present study was supported by the Grant PI11/00371 from the Instituto de Investigacion Carlos III, Ministerio de Economia y Competitividad (Spain).

\section{References}

1. Hornebeck W, Emonard H, Monboisse JC and Bellon G: Matrix-directed regulation of pericellular proteolysis and tumor progression. Semin Cancer Biol 12: 231-241, 2002.

2. Wilkins-Port CE, Higgins SP, Higgins CE, Kobori-Hotchkiss I and Higgins PJ: Complex regulation of the pericellular proteolytic microenvironment during tumor progression and wound repair: functional interactions between the serine rrotease and matrix metalloproteinase cascades. Biochem Res Int 2012: 454368, 2012.

3. Netzel-Arnett S, Hooper JD, Szabo R, et al: Membrane anchored serine proteases: a rapidly expanding group of cell surface proteolytic enzymes with potential roles in cancer. Cancer Metastasis Rev 22: 237-258, 2003.

4. Lu D, Yuan X, Zheng X and Sadler JE: Bovine proenteropeptidase is activated by trypsin, and the specificity of enteropeptidase depends on the heavy chain. J Biol Chem 272: 31293-31300, 1997.

5. Gladysheva IP, Wang D, McNamee RA, et al: Corin overexpression improves cardiac function, heart failure, and survival in mice with dilated cardiomyopathy. Hypertension 61: 327-332, 2013.

6. Folgueras AR, de Lara FM, Pendas AM, et al: Membrane-bound serine protease matriptase-2 (Tmprss6) is an essential regulator of iron homeostasis. Blood 112: 2539-2545, 2008.

7. Guipponi M, Tan J, Cannon PZ, et al: Mice deficient for the type II transmembrane serine protease, TMPRSS1/hepsin, exhibit profound hearing loss. Am J Pathol 171: 608-616, 2007.

8. Wu Q: Type II transmembrane serine proteases. Curr Top Dev Biol 54: 167-206, 2003.

9. Webb SL, Sanders AJ, Mason MD and Jiang WG: Type II transmembrane serine protease (TTSP) deregulation in cancer. Front Biosci 16: 539-552, 2011.

10. Hashimoto T, Kato M, Shimomura $\mathrm{T}$ and Kitamura N: TMPRSS13, a type II transmembrane serine protease, is inhibited by hepatocyte growth factor activator inhibitor type 1 and activates pro-hepatocyte growth factor. FEBS J 277: 4888-4900, 2010 .
11. Wu Q and Parry G: Hepsin and prostate cancer. Front Biosci 12: 5052-5059, 2007

12. Chen CH, Su KY, Tao MH, et al: Decreased expressions of hepsin in human hepatocellular carcinomas. Liver Int 26: 774-780, 2006.

13. Szabo R and Bugge TH: Type II transmembrane serine proteases in development and disease. Int $\mathrm{J}$ Biochem Cell Biol 40: 1297-1316, 2008.

14. Kosa P, Szabo R, Molinolo AA and Bugge TH: Suppression of Tumorigenicity-14, encoding matriptase, is a critical suppressor of colitis and colitis-associated colon carcinogenesis. Oncogene 31: 3679-3695, 2012

15. Lang JC and Schuller DE: Differential expression of a novel serine protease homologue in squamous cell carcinoma of the head and neck. Br J Cancer 84: 237-243, 2001.

16. Viloria CG, Peinado JR, Astudillo A, et al: Human DESC1 serine protease confers tumorigenic properties to MDCK cells and it is upregulated in tumours of different origin. Br J Cancer 97: 201-209, 2007.

17. Cal S, Quesada V, Garabaya C and Lopez-Otin C: Polyserase-I, a human polyprotease with the ability to generate independent serine protease domains from a single translation product. Proc Natl Acad Sci USA 100: 9185-9190, 2003.

18. Okumura Y, Hayama M, Takahashi E, et al: Serase-1B, a new splice variant of polyserase-1/TMPRSS9, activates urokinasetype plasminogen activator and the proteolytic activation is negatively regulated by glycosaminoglycans. Biochem J 400: 551-561, 2006.

19. Cantero D, Friess H, Deflorin J, et al: Enhanced expression of urokinase plasminogen activator and its receptor in pancreatic carcinoma. Br J Cancer 75: 388-395, 1997.

20. Wallrapp C, Hahnel S, Muller-Pillasch F, et al: A novel transmembrane serine protease (TMPRSS3) overexpressed in pancreatic cancer. Cancer Res 60: 2602-2606, 2000.

21. Uhland K, Siphos B, Arkona C, et al: Use of IHC and newly designed matriptase inhibitors to elucidate the role of matriptase in pancreatic ductal adenocarcinoma. Int J Oncol 35: 347-357, 2009.

22. Kilpatrick LM, Harris RL, Owen KA, et al: Initiation of plasminogen activation on the surface of monocytes expressing the type II transmembrane serine protease matriptase. Blood 108: 2616-2623, 2006

23. Min HJ, Lee Y, Zhao XF, et al: TMPRSS4 upregulates uPA gene expression through JNK signaling activation to induce cancer cell invasion. Cell Signal 26: 398-408, 2013.

24. Deer EL, Gonzalez-Hernandez J, Coursen JD, et al: Phenotype and genotype of pancreatic cancer cell lines. Pancreas 39: 425-435, 2010.

25. Vila MR, Lloreta J, Schussler MH, Berrozpe G, Welt S and Real FX: New pancreas cancers cell lines that represent distinct stages of ductal differentiation. Lab Invest 72: 395-404, 1995.

26. Noel A, Gutierrez-Fernandez A, Sounni NE, et al: New and paradoxical roles of matrix metalloproteinases in the tumor microenvironment. Front Pharmacol 3: 140, 2012.

27. Ochi N, Tanasanvimon S, Matsuo Y, et al: Protein kinase D1 promotes anchorage-independent growth, invasion, and angiogenesis by human pancreatic cancer cells. J Cell Physiol 226: 1074-1081, 2011.

28. Guo XZ, Xu JH, Liu MP, et al: KAI1 inhibits anchorage-dependent and -independent pancreatic cancer cell growth. Oncol Rep 14: $59-63,2005$.

29. Suzuki M, Kobayashi H, Kanayama N, et al: Inhibition of tumor invasion by genomic down-regulation of matriptase through suppression of activation of receptor-bound pro-urokinase. J Biol Chem 279: 14899-14908, 2004.

30. Liu J, Ben QW, Yao WY, et al: BMP2 induces PANC-1 cell invasion by MMP-2 overexpression through ROS and ERK. Front Biosci 17: 2541-2549, 2012.

31. Touab M, Villena J, Barranco C, Arumi-Uria M and Bassols A: Versican is differentially expressed in human melanoma and may play a role in tumor development. Am J Pathol 160: 549-557, 2002.

32. Welman A, Sproul D, Mullen P, et al: Diversity of matriptase expression level and function in breast cancer. PLoS One 7: e34182, 2012.

33. Jung H, Lee KP, Park SJ, et al: TMPRSS4 promotes invasion, migration and metastasis of human tumor cells by facilitating an epithelial-mesenchymal transition. Oncogene 27: 2635-2647, 2008. 